\title{
The Preventative Effect of $\alpha$-Tocopherol on Spinal Epidural Fibrosis After Laminectomy in a Rat Model
}

\author{
Cengiz TUNCER ${ }^{1}$, Canan SUBASI ${ }^{1}$, Cem DINC ${ }^{1}$, Erhan TURKOGLU², Uygur ER ${ }^{1}$ \\ ${ }^{1}$ Duzce University, School of Medicine, Department of Neurosurgery, Duzce, Turkey \\ ${ }^{2}$ Yildirim Beyazit Training and Research Hospital, Neurosurgery Clinic, Ankara, Turkey
}

\section{ABSTRACT}

AIM: To assess the efficacy of a-tocopherol as a topical antifibrotic agent on epidural fibrosis in lumbar laminectomy procedures.

MATERIAL and METHODS: A total of 32 adult rats were assigned into four groups, with eight rats in each group. Standard laminectomy was performed in every rat. In group 1, no treatment was administered after laminectomy. In group 2, a saline-soaked absorbable gelatin sponge was left on the dura mater after laminectomy. In group 3, only a-tocopherol was used on the dura mater after laminectomy. In group 4, a gelatin sponge soaked with a-tocopherol was used after laminectomy. En bloc vertebral columns between T10 and L5 were removed after sacrifice on postoperative day 30. Specimens were evaluated for degree of fibrosis and arachnoidal invasions.

RESULTS: Statistically significant differences were found in the mean of fibrosis grades between groups ( $p=0.001)$. Differences in mean arachnoidal invasion were not statistically significant $(p>0.05)$.

CONCLUSION: Topical application of a-tocopherol could effectively reduce the development of epidural fibrosis in an experimental laminectomy model.

KEYWORDS: a-tocopherol, Laminectomy model, Postlaminectomy fibrosis, Vitamin E

\section{INTRODUCTION}

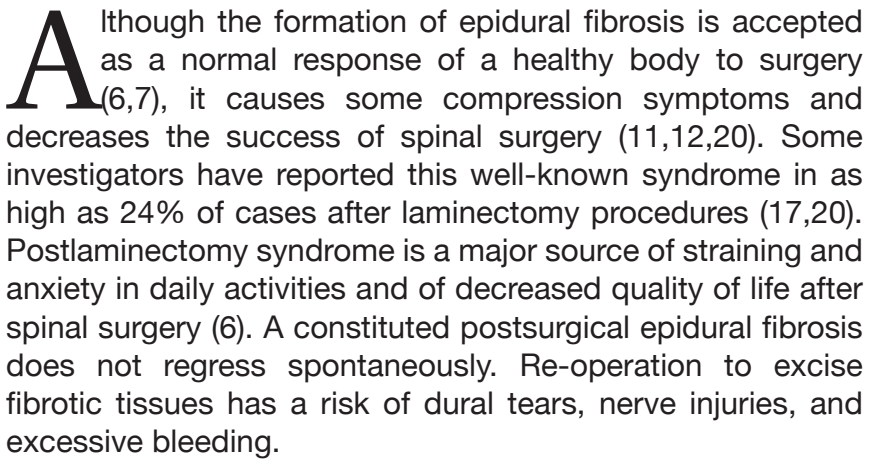

Preventing epidural fibrosis appears to be a logical to avoid such complications of re-operation. Methods and chemicals used to prevent epidural fibrosis are numerous in the literature. Biological and non-biological constituents to prevent epidural fibrosis have been studied in several experiments $(10,13)$.

In the current literature, to our knowledge, there is no clinical or experimental study on the efficacy of a-tocopherol (a-TCP) on epidural fibrosis after spinal operations. Therefore, this study was assessed the efficacy of a-TCP as a topical antifibrotic agent in the development of epidural fibrosis after lumbar laminectomy procedures. The main reason for choosing a-TCP to test antifibrotic activity is that it is one of the most potent antioxidants in humans and has an apoptotic effect on some cell lines that are considered to have a role in epidural fibrosis development $(5,18,19)$. 


\section{MATERIAL and METHODS}

Ethical approval of the study was received from the Committee of Ethics for Animal Studies of Abant Izzet Baysal University on April 14, 2014 (\#2013/12). The study was conducted at Duzce University.

\section{Animals}

A total of 32 adult female Wistar rats weighing 250 to 350 $\mathrm{g}$ were used in this study. Maximum effort was expended to minimize discomfort of the animals during surgery and sacrifice.

\section{Surgical Intervention and Taking Samples}

Every rat underwent the same surgical procedure. General anesthesia was induced by intraperitoneal xylazine $(10 \mathrm{mg} /$ kg; Bayer, İstanbul, Turkey) and ketamine hydrochloride (60 $\mathrm{mg} / \mathrm{kg}$; Parke-Davis, İstanbul, Turkey). After the low back area of each rat was shaved, the surgical site was sterilized with povidone. A longitudinal midline skin incision was made between the L2 and L5 spinous processes. The lumbosacral fascia was opened longitudinally and the paraspinous muscles were dissected bilaterally in a subperiosteal fashion to expose the laminae of L3-L5. L3-L5 laminectomy and flavectomy were accomplished, and epidural fat was removed, leaving the dura mater clean and dry. Hemostasis was achieved using cotton pads. No cautery or bipolar coagulation was used. After laminectomy, the wounds were closed in anatomical fashion via propylene suture (Prolene polypropylene sutures; Ethicon; Ethicon Endo-Surgery, Inc., Cincinnati, OH, USA). There were no complications or adverse effects due to materials used. The animals were sacrificed on postoperative day 30 using a lethal dose $(60 \mathrm{mg} / \mathrm{kg}$ ) of pentobarbital (IE Ulagay, Istanbul, Turkey). En-bloc vertebral columns between T10 and L5 were removed after sacrifice and placed into the $10 \%$ buffered formalin.

\section{Groups}

The 32 rats were allocated into four groups randomly, with eight rats in each group:

In group 1 (control group) no treatment was administered after laminectomy, and the wound was closed after achieving hemostasis.

In group 2, a $0.5 \mathrm{~mL}$ saline-soaked absorbable gelatin sponge $2 \mathrm{~mm}$ thick (Surgifoam 100-C Ethicon; Ethicon Endo-Surgery, Inc.) was left on the dura mater after laminectomy.

In group 3, only a-TCP acetate $0.5 \mathrm{~mL}$ was placed on the dura mater after laminectomy using an injector after meticulous hemostasis was achieved and the wound was closed without suction.

In group 4, a gelatin sponge $2 \mathrm{~mm}$ thick was soaked in 0.5 $\mathrm{mL}$ a-TCP $\left(\mathrm{C}_{29} \mathrm{H}_{50} \mathrm{O}_{2}\right)$ acetate (BASF Chemical Company, Ludwigshafen, Germany, Lot No:11076736WO).

\section{Assessment and Grading of Epidural Fibrosis}

The specimens were decalcified in ethylenediamine tetraacetic acid solution (EDTA; R\&D Systems, Inc., Minneapolis, MN,
USA), dehydrated, and embedded in paraffin blocks after decalcification was completed. Sections of $10 \mu \mathrm{m}$ were obtained axially, stained with Masson's trichrome, and examined using a microscope (Eclipse 80i; Nikon, Tokyo, Japan) and photographed using a camera (Nikon DS- Fi1). All laminectomy samples were evaluated by one pathologist in a blinded fashion, who analyzed the dural thickness, density of fibrosis, and arachnoid involvement. Quantitative morphometric analysis was performed on sections using the Nikon Nis Elements D 3.1 Digital Analyzing System. Measurements were performed under a magnification of $\times 100$.

The dura mater thickness was measured at three points. The first sample was harvested from the midpoint of the laminectomy defect, the second $2 \mathrm{~mm}$ from the right side of the first sample, and the third $2 \mathrm{~mm}$ from the left side of the first sample, as defined and performed by Cemil et al.(6). Epidural fibrosis was graded based on the scheme developed by $\mathrm{He}$ et al.(11). Mean values were used for statistical evaluation. Grading of fibrosis was designated as follows:

Grade 0: Dura mater was free of scar tissue.

Grade 1: Only thin fibrous bands were observed between the scar tissue and dura mater.

Grade 2: Continuous adherence was observed in less than two-thirds of the laminectomy defect.

Grade 3: Scar tissue adherence was extensive, affecting more than two-thirds of the laminectomy defect, or the adherence extended to the nerve roots. The presence of arachnoidal involvement also was noted.

\section{Statistical Analysis}

The data were analyzed using statistical software (SPSS, version 20.0 for Windows; SPSS, Inc., Chicago, IL, USA). Descriptive statistics for ordinal variables are represented as $25^{\text {th }}$ to $75^{\text {th }}$ percentiles, and categorical variables are represented as numbers of rats and percentages. To evaluate percentile differences among groups, the $x^{2}$ test was used. If the expected values were $<5$ in boxes, Fisher's exact test was used. To determine statistical significance between groups regarding the density of epidural fibrosis, the Kruskal-Wallis test was used. If the Kruskal-Wallis test was statistically significant, Conover's non-parametric multiple comparison test was used to determine the situations that caused the differences. The presence of arachnoidal involvement was analyzed statistically using a likelihood ratio test. $p$ values $<0.05$ were accepted as statistically significant.

\section{RESULTS}

There was no loss of rats related to the procedure, and all animals were ambulatory at the time of sacrifice. No wound infection, erythema, hematoma, or cerebrospinal fluid leakage was observed. Fibrosis and arachnoidal invasion rates in the groups are shown in Tables I and II, respectively. Statistically significant differences were found in the mean of fibrosis grades among the groups ( $p=0.001$; Figure 1). Group 4 had the best results because of the epidural fibrosis grades and group 1 had the lowest score. Differences between groups 
were statistically significant $(\mathrm{p}<0.005$; Table III). Differences in mean of arachnoidal invasion between the groups were not statistically significant ( $p>0.05)$. However, arachnoidal invasion was less in group 4 than the other groups. In the axial sections stained with Masson's trichrome, $75 \%$ of the rats in group 1 showed grade 3 epidural fibrosis (Figure 2). A sample from group 3 demonstrated grade 2 epidural fibrosis (Figure 3 ) with the same staining and magnification.

Table I: Fibrosis Grades of Groups

\begin{tabular}{cccc}
\hline Group & Fibrosis Grade & $\mathbf{n}$ & $\%$ \\
\hline \multirow{2}{*}{ I } & 2 & 2 & 25.0 \\
& 3 & 6 & 75.0 \\
\hline \multirow{3}{*}{ II } & 1 & 2 & 25.0 \\
& 2 & 5 & 62.5 \\
& 3 & 1 & 12.5 \\
\hline \multirow{2}{*}{ III } & 1 & 1 & 12.5 \\
& 2 & 4 & 50.0 \\
\multirow{2}{*}{ IV } & 3 & 3 & 37.5 \\
& 1 & 2 & 25.0 \\
& 2 & 6 & 75.0 \\
\hline
\end{tabular}

Table II: Arachnoidal Invasion of Groups

\begin{tabular}{cccc}
\hline Group & & $\mathbf{n}$ & $\%$ \\
\hline I & $(-)$ & 5 & 62.5 \\
\hline & $(+)$ & 3 & 37.5 \\
\hline & Total & 8 & 100.0 \\
\hline II & $(-)$ & 7 & 87.5 \\
\hline & $(+)$ & 1 & 12.5 \\
\hline & Total & 8 & 100.0 \\
\hline III & $(-)$ & 6 & 75.0 \\
\hline & $(+)$ & 2 & 25.0 \\
\hline & Total & 8 & 100.0 \\
\hline IV & $(-)$ & 8 & 100.0 \\
\hline
\end{tabular}

Table III: Differences Between Groups according to Fibrosis Grades ( $p$ values*)

\begin{tabular}{cccccc}
\hline Groups & $\%$ F & I & II & III & IV \\
\hline I & 11.25 & - & $<0.005$ & $<0.05$ & $<0.001$ \\
\hline II & 12.94 & $<0.005$ & - & $<0.05$ & $<0.005$ \\
\hline III & 17.69 & $<0.05$ & $<0.05$ & - & $<0.005$ \\
\hline IV & 24.13 & $<0.001$ & $<0.005$ & $<0.005$ & - \\
\hline
\end{tabular}

${ }^{*}$ Calculations were performed by the Kruskal-Wallis test.

$\% \boldsymbol{F}=$ mean values of fibrosis grades.

\section{DISCUSSION}

Epidural fibrosis is seen widely after laminectomy procedures. Some well-known surgical conditions have vital roles in the pathogenesis of this entity, such as amount of postoperative hematoma, development of postoperative infection, laminectomy techniques, amount of bone removed, and anatomic region of the spinal column (15).

Tumor necrosis factor-a (TNF-a), as one of the factors responsible for epidural fibrosis, inhibits fibrillary collagen gene expression and stimulates cytokines and interstitial collagenase, or in other words matrix metalloproteinase (MMP) gene expression (12). TNF-a is a potent activator of the intracellular signaling molecules, and has a role in apoptosis, like cJun $\mathrm{N}$-terminal kinase (JNK or SAPK), p38

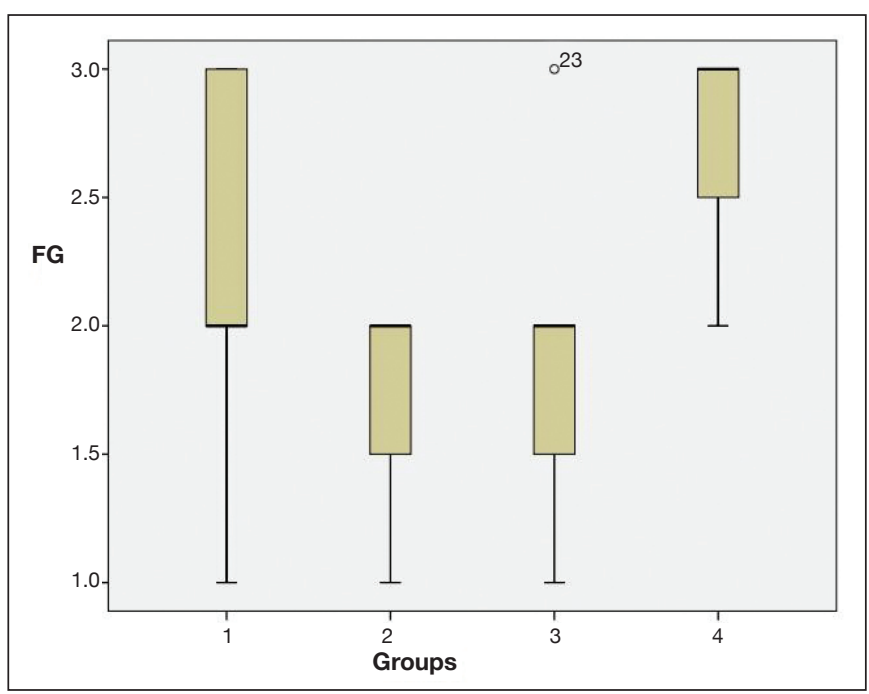

Figure 1: Box plot shows comparison of mean fibrosis grades (FG).

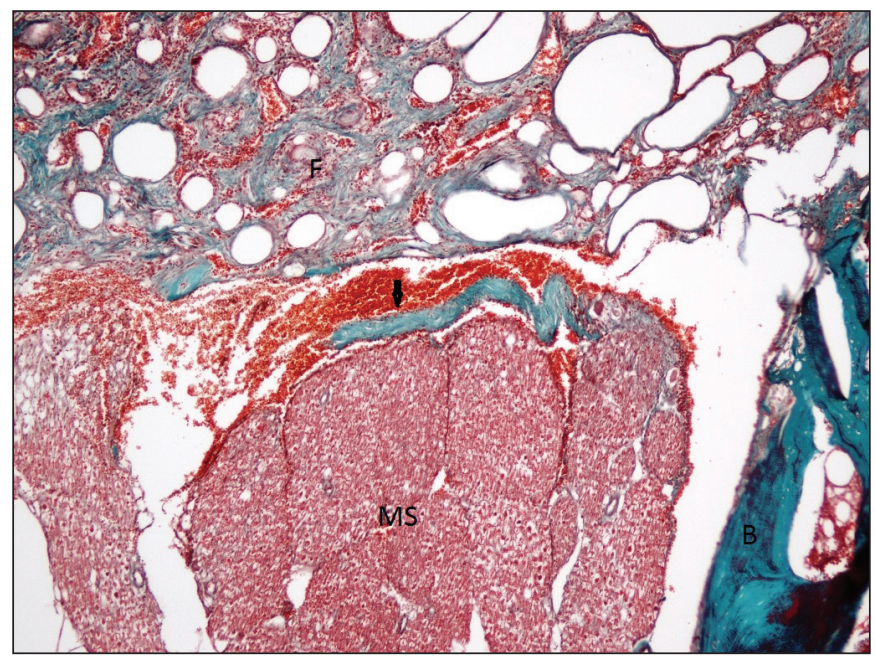

Figure 2: Photomicrograph shows "grade 3" fibrosis in group 1 (control group). Epidural fibrosis (F) completely covered the laminectomy defect and adhered to the underlying dura mater (arrow). Direct contact was evident between the epidural fibrosis and medulla spinalis (MS). (B: bone)(Masson's trichrome, $\times 100$ ). 
Tuncer C. et al: $\alpha$-Tocopherol and Epidural Fibrosis

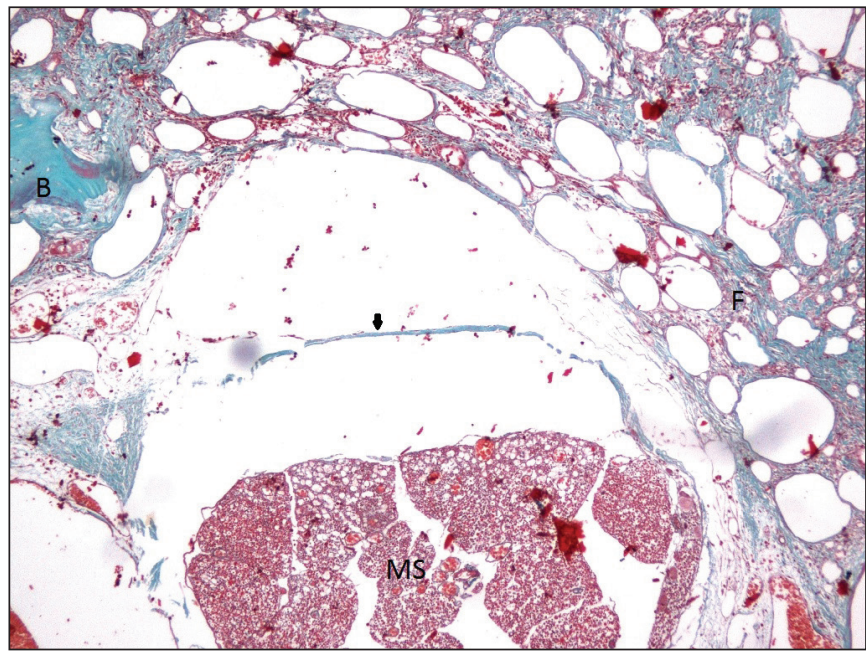

Figure 3: Photomicrograph shows "grade 2" fibrosis in group 3 (a-TCP group). Epidural fibrosis covered less than one-third of the laminectomy defect (F: Fibrosis, B: Bone, MS: Medulla spinalis) (Masson's trichrome, $\times 100$ ).

MAPK, and NF-KB. These substances control expression of type 1 collagen $\mathrm{a} 1$, interstitial collagenase, and cytokines, individually. TNF- $a$ increases the release of endothelin-1 and expression of adhesion molecules, such as E-selectin, SD54, and SD106, from endothelial cells. Moreover, it excites proliferation of fibroblasts via transforming growth factor (TGF)- $\beta 1$ (12). When tissue damage occurs, TGF- $\beta 1$ is excreted as a result of degranulation, and it behaves as a chemoattractant for inflammatory cells and thrombocytes, and stimulates fibroblasts, which are the main cells for developing fibrosis. The crucial element for the construction of the fibrosis is fibroblast migration into the damaged area (4). Fibroblasts originate from paravertebral muscles and are transported by blood into the damaged area. Consequently, inhibition of migration of fibroblasts from the paraspinal muscles or from hematoma related to surgery could prevent or reduce epidural fibrosis formation. Numerous biological, synthetic, or semisynthetic substances have been examined for the prevention of scar formation $(1,6,8,20)$ via direct inhibition on fibroblast migration.

Some research has found that the vital role of TNF-a in collagen synthesis and fibrosis could be antagonized and/ or reduced by the action of particular agents. The substance a-TCP might block the development of fibrosis not only by its direct effects on fibroblasts but also by decreasing TGF- $\beta$ levels (21). a-TCP has been hypothesized to be potentially beneficial in the mitigation of postlaminectomy epidural fibrosis in at least two ways: inhibition of direct fibroblast migration and decreasing TGF- $\beta$ levels. At the same time, a-TCP serves as a natural antioxidant. This effect of $\alpha-T C P$ may be because of its neuromediator effects on intracellular pathways (2). Another mechanism of action of a-TCP is the apoptotic effect on neoplastic, fibrotic, or normal cell lines. However, this apoptotic effect varies depending on cell type; it was exerted via the death receptor 5 and Cyt C-dependent mitochondrial pathways $(9,22)$. Moreover, the fact that lipid peroxidation-dependent proapoptotic Bax protein upregulation is maintained as responsible for this effect in neoplastic and normal cells also has been reported (16). These mechanisms indicate the importance of the mitochondrial pathway in apoptotic effects of a-TCP and also the possibility that Cyt c-dependent pathways may be used. Apoptotic effects of a-TCP on different cell lines attenuated some more experimental studies in the literature (14).

Histopathological evaluation was performed with Masson's trichrome staining. This staining method is used mainly to show the presence of collagen and muscle. It also can show increases in collagenous matter or to fibrotic changes in cirrhosis of the liver, renal and pulmonary diseases, and wound healing processes (3). In this study, fibrosis secondary to laminectomy alone was illustrated perfectly in all specimens. Microscopic examination demonstrated grade 3 fibrosis. Scar tissue with formation of dense epidural fibrosis was extensive, and its adherence covered more than two-thirds of the laminar defect; at the same time the adherence extended to the nerve roots. The presence of arachnoidal involvement was also noted. The tissue and significant adhesion were demonstrated in all group 1 specimens. This phenomenon was evaluated as "grade 3" epidural fibrosis in all group 1 specimens. Infections can cause grade 3 epidural fibrosis, but microscopic studies of the tissue revealed no sign of infection or chronic inflammation related to the surgical procedure. Microscopic examinations of group 4 specimens revealed slight epidural fibrosis, such as grade 1 or rarely 2 , and the results of these two groups were statistically significantly different $(p<0.005)$. This slight epidural fibrosis was confirmed using Masson's trichrome staining. This effect could not be due to a-TCP only, because gelatin sponges were used with a-TCP. This effect may have belonged to the gelatin sponge. To test this, groups 2 and 3 were designed in the study, where group 2 specimens were treated by only with the gelatin sponge and group 3 specimens were administered a-TCP topically by an injector. Specimens in group 2 showed mainly grade 2 epidural fibrosis microscopically. The degree of epidural fibrosis in group 2 was between that of groups 1 and 4 . The difference between groups 1 and 2 was statistically significant $(p<0.01)$. The epidural fibrosis level was statistically lower in group 2 than in group 1. However, this decrease was not as great as that in group 4 . There also was a statistically significant difference in epidural fibrosis level between groups 2 and $3(p<0.01)$.

The epidural fibrosis levels in group 4 were statistically lower than those in groups $1(p<0.005), 2(p<0.01)$, and $3(p<0.01)$. Results in group 3 were significantly different from those in groups 1 and $4(p<0.005)$ but were not different from those in group 2 ( $p>0.05)$. This statistical outcome demonstrated that the effect of reducing epidural fibrosis was not due to the gelatin sponge but to a-TCP because the epidural fibrosis was significantly less in groups 2 and 4 than in groups 1 and 3 $(p<0.005$ and $p<0.05$, respectively).

The comparison of arachnoidal involvement exhibited no statistically significant difference between the groups ( $p>0.05)$, although arachnoidal involvement was less in group 3 than in group 1. The mechanism of the development of arachnoidal 
adhesions was different than the fibrosis. Arachnoidal adhesion level and epidural fibrosis grade were not correlated based on the results of this study.

According to our results, a-TCP had an antifibrotic effect during the postoperative course of wound healing. Epidural hemostasis performed after laminectomy using a gelatin sponge also had an antifibrotic effect in the postoperative period.

In this study, we aimed to reveal a possible antifibrotic effect of a-TCP in reducing epidural fibrosis. This may be secondary to direct inhibition of TNF-a or fibroblast migration. It may also decrease TGF- $\beta$ levels (21). An apoptotic effect of a-TCP on fibroblasts is another possible mechanism. Mitochondrial pathways of this effect should be studied, and further experimental studies should be designed.

\section{Limitations of the Study}

In vivo and in vitro studies, including dose-response evaluations, are needed to verify these effects and draw solid conclusions regarding better use of a-TCP. In this study, dose standardization was the main problem. Groups 3 and 4 were administered $0.5 \mathrm{~mL}$ a-TCP, but this dose is not at a standardized level. Second, the exact mechanism of action, especially at the intracellular level, should be clarified. Surgical and cellular aspects are needed. Third, some antifibrotic combinations including $\mathrm{a}-\mathrm{TCP}$ are required to test better results. Sacrificing time is another limitation of the study. Epidural fibrosis level may be higher after a long-time period. Finally, the number of rats in the groups should be increased to augment the statistical power of the results.

\section{CONCLUSION}

Topical application of a-TCP could effectively reduce the development of epidural fibrosis in an experimental laminectomy model. Further research is necessary to define the optimal dosage and determine the safe dose limits of a-TCP.

\section{- REFERENCES}

1. Aydincak O, Yilmaz MB, Emmez H, Kurt G, Sepici A, Memis L, Baykaner K: The effect of temozolomide on the prevention of epidural fibrosis developing after lumbar laminectomy in rats. Turk Neurosurg 22(6):706-711, 2012

2. Azzi A, Stocker A: Vitamin E: Non-antioxidant roles. Prog Lipid Res 39:231-255, 2000

3. Bancroft J, Gamble M: Theory and Practice of Histological Techniques, 6th ed. London: Churchill-Livingstone, 2008

4. Border W, Noble N: Transforming growth factor beta in tissue fibrosis. N Engl J Med 11:1286-1292, 1994

5. Brigelius-Flohe R, Traber MG: Vitamin E: Function and metabolism. FASEB J 13:1145-1155, 1999

6. Cemil B, Tun K, Kaptanoglu E, Kaymaz F, Cevirgen B, Comert A, Tekdemir I: Use of pimecrolimus to prevent epidural fibrosis in a postlaminectomy rat model. J Neurosurg Spine 11(6):758763, 2009
7. Dogulu F, Kurt G, Emmez H, Erdem O, Memis L, Baykaner $\mathrm{K}$, Ceviker N: Topical mitomycin C-induced inhibition of postlaminectomy peridural fibrosis in rabbits. J Neurosurg 99(1):76-79, 2003

8. Einhaus SL, Robertson JT, Dohan FC Jr, Wujek JR, Ahmad S: Reduction of peridural fibrosis after lumbar laminotomy and discectomy in dogs by a resorbable gel (ADCON-L). Spine (Phila Pa 1976) 22(13):1440-1461,1997

9. Engin KN, Kuruca SE, Dar KA, Cetin B, Karadenizli S, Gurel E, Yemisci B, Bilgic S, Arslan M: The evaluation of human tenon's fibroblasts and endothelial cell responses to antifibrotics alone and in combination with a-Tocopherol. Curr Eye Res 40(1):19-29, 2015

10. Gill GG, Scheck M, Kelley ET, Rodrigo JJ: Pedicle fat grafts for the prevention of scar in low-back surgery. A preliminary report on the first 92 cases. Spine (Phila Pa 1976) 10(7):662667, 1985

11. HeY, Revel M, Loty B: A quantitative model of postlaminectomy scar formation. Effects of a nonsteroidal anti-inflammatory drug. Spine (Phila Pa 1976) 20(5):557-563, 1995

12. Ismailoglu O, Albayrak B, Gulsen I, Tanriover G, Demir N: Topical application of tacrolimus prevents epidural fibrosis in a rat postlaminectomy model: Histopathological and ultrastructural analysis. Turk Neurosurg 21(4):630-633, 2011

13. Jacobs RR, McClain O, Neff J: Control of postlaminectomy scar formation: An experimental and clinical study. Spine (Phila Pa 1976) 5(3):223-229, 1980

14. Jalil AA, Khaza'ai $H$, Nordin N, Mansor N, Zaulkffali AS: Vitamin E-mediated modulation of glutamate receptor expression in an oxidative stress model of neural cells derived from embryonic stem cell cultures. Evid Based Complement Alternat Med 2017:6048936, 2017

15. Kasimcan MO, Bakar B, Aktaş S, Alhan A, Yilmaz M: Effectiveness of the biophysical barriers on the peridural fibrosis of a postlaminectomy rat model: An experimental research. Injury 42(8):778-781, 2011

16. Khveuja KL, Kumar S, Varma N, Varma SC, Avti PK, Pathak $\mathrm{CM}$ : Enhancement in a-tocopherol succinateinduced apoptosis by all-trans-retinoic acid in primary leukemic cells: Role of antioxidant defense, Bax and c-myc. Mol Cell Biochem 319:133-139, 2008

17. Manchikanti L: Epidemiology of low back pain. Pain Physician 3:167-192, 2000

18. Nalecz K, Nalecz M, Azzi A: Isolation of tocopherol binding proteins from the cytosol of smooth muscle A7r5 cells. Eur J Biochem 209:37-42, 1992

19. Traber MG, Packer L: Vitamin E: Beyond antioxidant function. Am J Clin Nutr 62:1501-1509, 1995

20. Turkoglu E, Tuncer C, Dinc C, Sekerci Z: The effect of etanercept on spinal epidural fibrosis in a postlaminectomy rat model. Turk Neurosurg 24(4):506-511, 2014

21. Yamamoto T, Takagawa S, Katayama I, Nishioka K: Antisclerotic effect of transforming growth factor-beta antibody in a mouse model of bleomycin-induced scleroderma. Clin Immunol 92(1):6-13, 1999

22. Yu W, Park SK, Jia L, Tiwary R, Scott WW, Li J: RRR-gtocopherol induces human breast cancer cells to undergo apoptosis via death receptor 5 (DR5)-mediated apoptotic signaling. Cancer Lett 259:165-176, 2008 